\title{
CRYOCRYSTALLOGRAPHY PAPERS
}

Contributions intended for this section should be submitted to Dr P. C. Weber, Schering-Plough Research Institute, K-15-3/3855, 2015 Galloping Hill Road, Kenilworth, NJ 07033-0539, USA. They may be full research papers, short communications or brief notes. The International Union of Crystallography can assume no responsibility for the accuracy of the claims made.

J. Appl. Cryst. (1996). 29, 465-468

\section{Low-cost conversion of a coaxial nozzle arrangement into a stationary low-temperature attachment}

THOMAS KOTTKE, ${ }^{a} \dagger$ RICHARD J. LAGOW ${ }^{a}$ AND DIETMAR STALKE ${ }^{b} \dagger$ at ${ }^{a}$ Department of Chemistry and Biochemistry, University of Texas at Austin, Austin, TX 78712, USA, and ${ }^{b}$ Department of Inorganic Chemistry, University of Göttingen, Tammannstrasse 4, D-37077 Göttingen, Germany. E-mail: tkottke@xiris.chemie.uni-wuerzburg.de

(Received 22 December 1995; accepted 4 March 1996)

\begin{abstract}
A stationary nozzle accessory designed for the Siemens/Nicolet LTII low-temperature device with standard $\varphi$-coaxial nozzle arrangement is described. The attachment is easy to assemble and its compatibility allows interchanging between both systems in less than $1 \mathrm{~h}$, if required. Implementation of the stationary nozzle reduces the consumption of liquid nitrogen by more than $40 \%$ and extends the temperature range available for data collection by $60 \mathrm{~K}$ in the lower temperature range. Details of construction are presented, and a comparison with the $\varphi$ coaxial nozzle is discussed.
\end{abstract}

\section{Introduction}

The application of low-temperature techniques has become an invaluable method to considerably improve the quality of a crystal structure analysis (Rudman, 1976; Veith \& Frank, 1988; Luger, 1989). Numerous solid-state investigations of single crystals unstable at ambient temperature have been reported to date. Cooling the specimen during data collection significantly reduces the thermal motion of atoms (Brock \& Dunitz, 1990). The electron-density map is notably higher resolved, facilitating the location of $\mathrm{H}$-atom positions and electron-deformation density studies (Koritsanszky, Buschmann, Luger, Knochel \& Patz, 1994). Nowadays, a variety of low-temperature attachments based on gas-stream cooling are commercially available (Cosier \& Glazer, 1986; Stoe Diffraction Systems, 1987; EnrafNonius, MSC/Rigaku and Siemens also offer low-temperature attachments).

While the generation of the cold gas stream via liquid nitrogen boil-off prevails (Veith \& Frank, 1988), different principles are employed to direct the gas flow towards the crystal. Two general concepts can be classified with regard to the orientation of the low-temperature nozzle. The $(\varphi)$ coaxial nozzle is arrayed in line with the $\varphi$ axis such that the nitrogen stream follows the motion of the goniometer-head axis. This arrangement is realized by mounting of the nozzle directly on the $\chi$ circle, which requires a flexible construction of the transfer line, e.g. a combination of glass tubes connected via swivel joints (Strouse, 1976). The stationary nozzle directs the cold gas from a fixed position towards the crystal. As this construction is completely separated from the goniometer, an

† Now at Department of Inorganic Chemistry, University of Würzburg, Am Hubland, D-97074 Würzburg, Germany. orientation of the nozzle can be chosen that minimizes the extent to which the versatility of the diffractometer is limited.

If the conventional technique of sealing the specimen in a capillary is applied, the coaxial nozzle arrangement ensures the smallest possible temperature variation along the capillary throughout all goniometer movements. On the other hand, mounting the crystal on the tip of a glass fibre merely requires a laminar flow of the gas stream to achieve temperature uniformity at the crystal. This can be accomplished equally well by both systems. Owing to the lack of moving parts, no 'cooling loss' occurs, and a significantly lower temperature at the crystal position as well as a lower consumption of liquid nitrogen is obtained with the stationary nozzle. Moreover, the risk of glass breakage resulting from ice formation inside the swivel joints of the coaxial system does not apply to a fixed arrangement. We describe here a low-cost and easy-to-assemble stationary nozzle accessory designed for the Siemens/Nicolet LTII low-temperature attachment. Its implementation requires only minor alterations of the standard device, permitting the employment of the coaxial system at any time.

\section{Description}

The stationary nozzle accessory consists of a vacuum-jacketed glass tube with a $45^{\circ}$ transfer-line extension part and a Teflonbased dual stream nozzle (Fig. 1). A firm transition to the metal transfer line is accomplished by the placing of a Tefion spacer into the Teflon adapter insert chamber of the glass tube. The connection is sealed with a rubber boot taken from the LTII device and secured by two tension springs. In addition, a lightweight clamp mounted on the collimator supports the lower part of the glass tube, facilitating the adjustment of the nozzle position. Vibration of the framework, particularly during liquidnitrogen refill, is suppressed by support of the metal transfer line through a normal laboratory rack anchored to the diffractometer base. As the metal transfer line is shifted by approximately $260 \mathrm{~mm}$ towards the X-ray tube, the rack can be conveniently accommodated in the area behind the tube housing. The orientation of the nozzle causes the nitrogen stream to reach the crystal at a $45^{\circ}$ angle with respect to the reflection plane. In contrast to a coaxial nozzle, this arrangement does not require the use of a deflector to protect the goniometer head, i.e. it resembles an offset position of the coaxial nozzle.

Details of the glass transfer line are given in Fig. 2. After completion of the glassblowing work, the inside is silvered and 
then evacuated for at least a day before sealing off. As this step determines the quality of the thermo-insulation, the entire glass tube should be maintained at $\geq 432 \mathrm{~K}$ during evacuation and a diffusion pump should be used. Owing to manufacturing tolerances in the dimensions of the glass tubes, the Teflon adapter has to be adjusted to the completed transfer line. The length of the spacer should be 1-2 mm less than the depth of the insert chamber to ensure a proper fit of the rubber boot.

A Teflon segment with circular cross-section (Fig. 3) forms the base of the dual-stream nozzle (Stalke, 1987; Kottke, 1993). One end slides over the insert of the glass transfer line while the other end hosts the stainless-steel nozzle tip with integrated heating coil. A common soda (drinking) straw, centered by six Teflon screws with stainless-steel needle tips, forms the inner tube separating the cold gas stream from the outer warm gas shell. Unlike techniques involving two individual gas-stream generators or an additional dry gas supply regulated by (often unreliable) needle valves, this simple method for producing a distinct warm gas envelope guarantees a gas flow free of any turbulence. Furthermore, the difference in velocity between the inner and the outer streams causes a significant extension of the cold-gas zone. In comparison with a conventional (conical) single-chamber nozzle, where the nozzle tip is electrically heated as well, the dual-stream nozzle ensures that the central

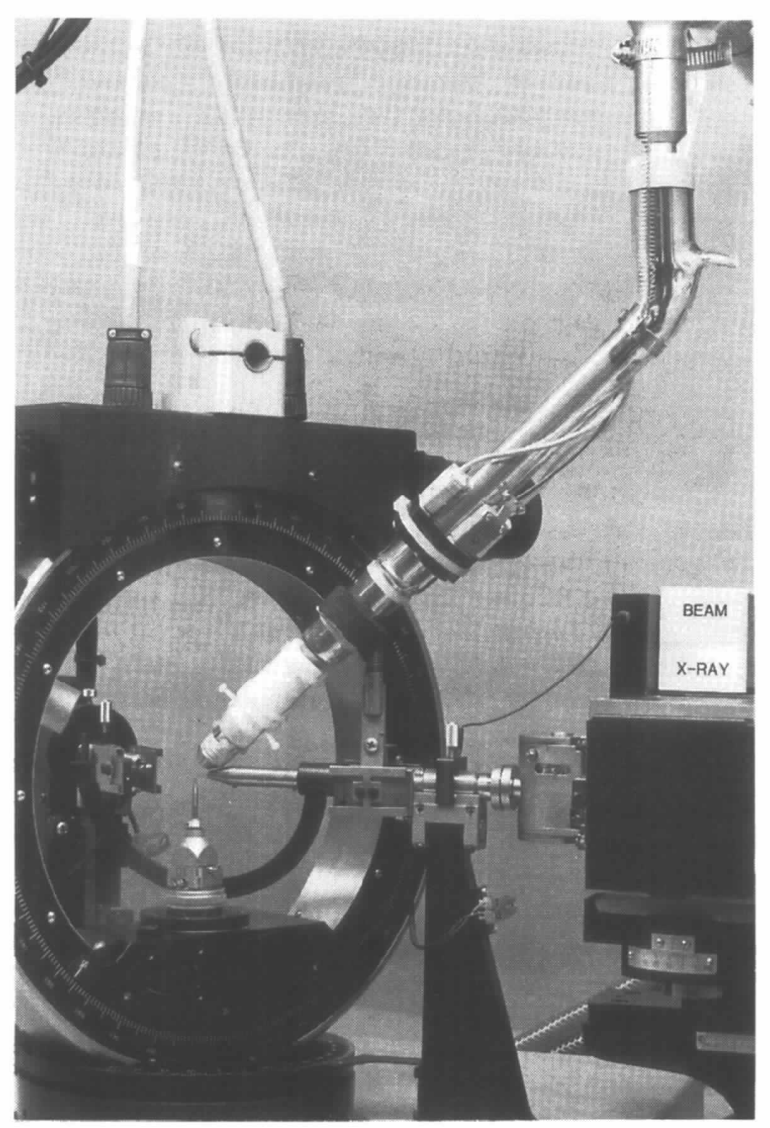

Fig. 1. The stationary nozzle attachment connected to the LTII lowtemperature device. The transition from the metal transfer line to the glass transfer line is located in the upper right corner of the picture. gas stream is unaffected by the outer stream, independent of the gas-flow rate.

The thermocouple for temperature monitoring and control is led through a $0.3 \mathrm{~mm}$ drill hole in the Teflon cover (Fig. 3) and centered in the cold gas stream. The nozzle heater can be directly connected to the nozzle-heater power supply. Insertion of a nozzle-heater controller, however, ensures a constant temperature of the enveloping warm gas stream independent of the flow rate and protects the nozzle against overheating even in the case of a transfer-line freeze-up. A suitable circuit diagram for a commercially available temperature-control unit is shown in Fig. 4. As the solid-state relay requires a constant-voltage AC power supply, the nozzle heater of the LTII control module has to be set to maximum output to switch off pulsing. Depending on the desired design, the cost of materials for the complete accessory amounts to approximately US $\$ 200$, or US $\$ 500$ if a nozzle-heater temperature controller is included.

\section{Capabilities and experience}

With application of the oil-drop mounting technique pioneered by Hope $(1987,1988)$, the stationary nozzle accessory has been in use for over seven years with a Stoe AED diffractometer, and for almost two years on a Siemens P4 diffractometer. Within the temperature range accessible by both the coaxial and the stationary attachment, the quality of the data set at a given

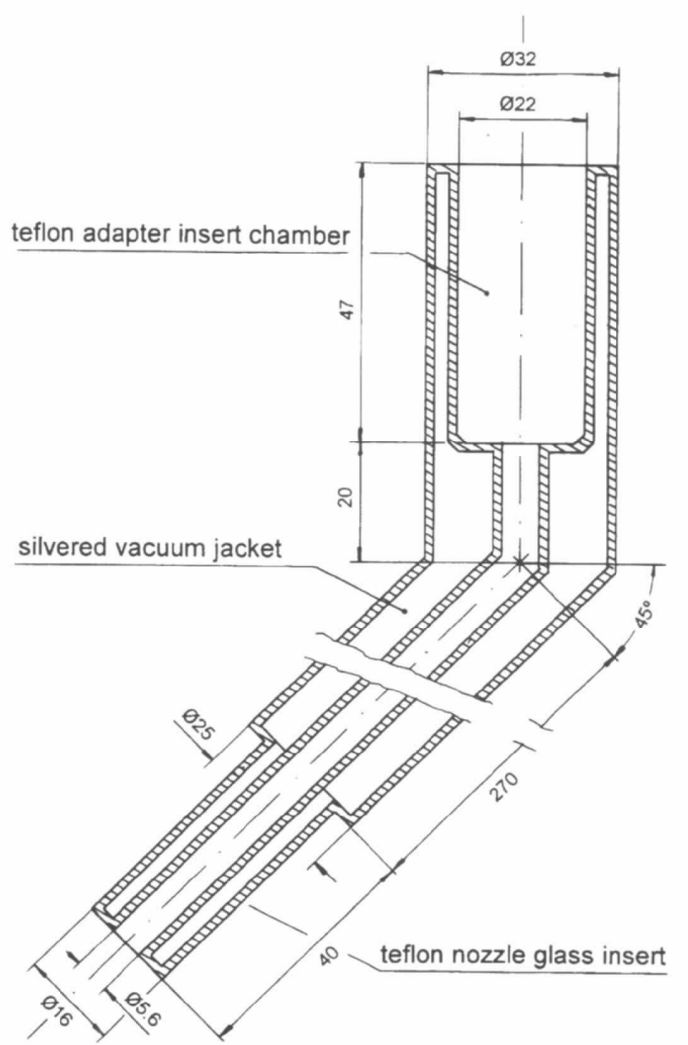

Fig. 2. The glass transfer line. The standard walled glass tubes utilized have outer diameters of $8,16,25$ and $32 \mathrm{~mm}$. Two glass hooks taking the contraction springs are placed opposite each other below the Teflon adapter insert chamber. 
Table 1. Technical data for the coaxial and the stationary nozzle accessory

Temperature range $(\mathrm{K})$

$\mathrm{LN}_{2}$ consumption at $153 \mathrm{~K}\left(1 \mathrm{~d}^{-1}\right)$

Angular range in $\chi\left(^{\circ}\right)$

\begin{tabular}{cc}
\multicolumn{2}{c}{ Nozzle arrangement } \\
\hline Coaxial & Stationary \\
$153^{*}-213$ & $93-188$ \\
49 & 28 \\
$-90-75$ & $-120-120$
\end{tabular}

* The limitation of the minimum temperature is due to ice formation in the swivel joints.

temperature is not influenced by the choice of the system. With respect to efficiency and support of the normal diffractometer operation, however, the stationary nozzle is shown to have major advantages over the coaxial nozzle (Table 1). At $153 \mathrm{~K}$, the consumption of liquid nitrogen is reduced by more than $40 \%$. Comparative measurements performed without moving of the circles indicate an even more pronounced effect in the lower-temperature region. Because of ice formation inside the swivel joints, data collection at temperatures below $153 \mathrm{~K}$ was not attempted using the coaxial nozzle arrangement.

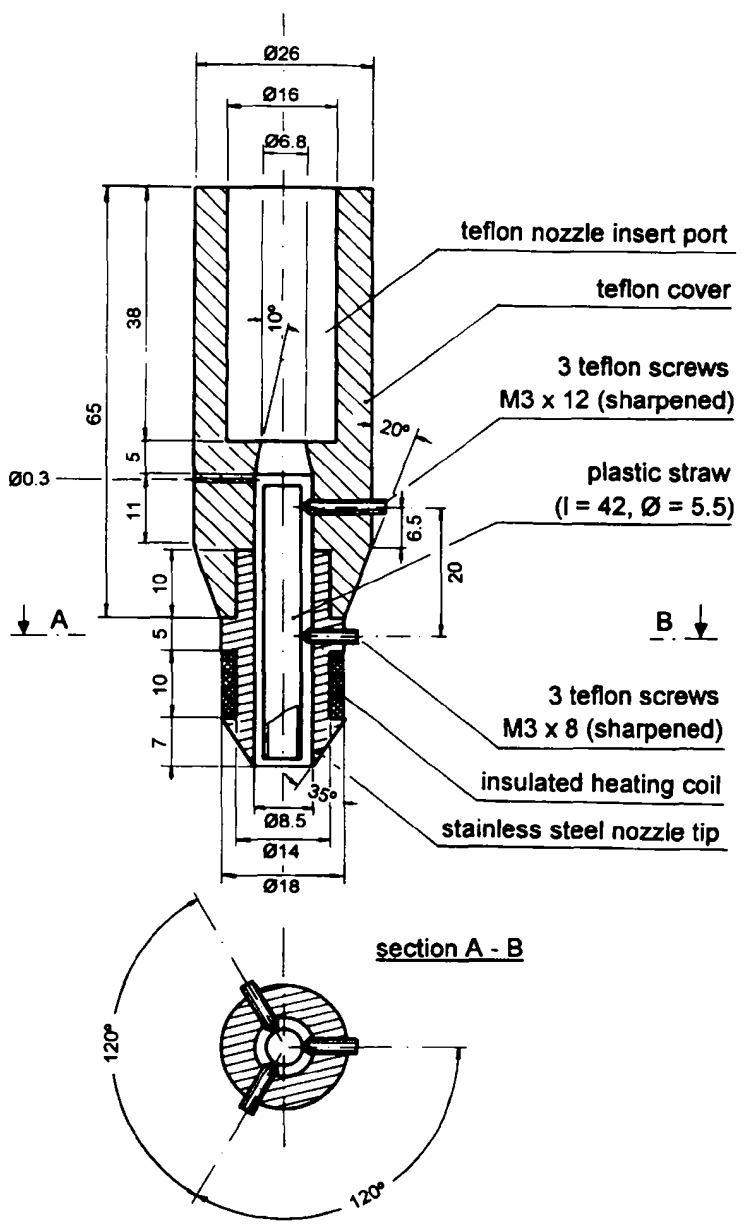

Fig. 3. The dual-stream low-temperature nozzle. Thermocouple: chromel-alumel, $\varnothing=0.25 \mathrm{~mm}$, connected to the LTII control module; nozzle heater: insulated heating wire, $R=20$.
The orientation of the stationary nozzle along the collimator not only simplifies the determination of angular restrictions but also reduces their extent. The accessible range of the $\chi$ circle now permits complete $\psi$ rotations, affording a considerably improved empirical absorption correction. When $\omega$ is close to zero, single $360^{\circ}$ rotations in $\chi$ can be executed, which is essential for accurate centering of the crystal height. Furthermore, the larger freedom in the goniometer region facilitates structure analyses of temperature-sensitive compounds (Kottke \& Stalke, 1993). Implementation of a dualstream rather than a single-stream nozzle allows the distance between the crystal and the nozzle outlet to be moderately varied without this affecting the temperature at the crystal position. This offers the possibility of even further decreasing the angular limitations of the diffractometer. On the other hand, precise centering of the inner cold gas stream with respect to the crystal is essential for the maintenance of a laminar gas stream throughout all goniometer movements. We experienced an excessive formation of ice on the crystal mount owing to slight misalignment of the nozzle. However, once the stationary nozzle was properly centered and secured, its position did not require readjustment for a long period of time.

There are several options for maintaining a frost-free environment of the crystal. Without any doubt, the best solution is to surround the entire diffractometer with an air-tight enclosure in which the humidity is reduced to $10 \%$ or less. This can be accomplished by permanent drying of the atmosphere inside the box and/or by forcing of dry air into the enclosure, which generates a positive pressure inside (Abowitz \& Ladell, 1968). For normal operation, a tapered copper tube $(\varnothing 6 \times \varnothing 0.8 \times$ length $30 \mathrm{~mm})$ forming the mounting pin in combination with the dual-stream nozzle can suppress the formation of ice reasonably well, even during exposures of several days. The enclosed glass fiber $(\varnothing 0.2 \mathrm{~mm})$ has to be accurately centered within the tube and well fixed using, for instance, two-component dental cement. When the glass fiber is cut to a length of $3 \mathrm{~mm}$ outside the copper sheath, the tip of the copper pin always projects into the warm gas stream, and the transition to the glass fiber remains free of ice. With the low-temperature nozzle precisely aligned, only gradual covering of the specimen with a thin film of ice occasionally occurs, which may be attributed to the high humidity in Texas.

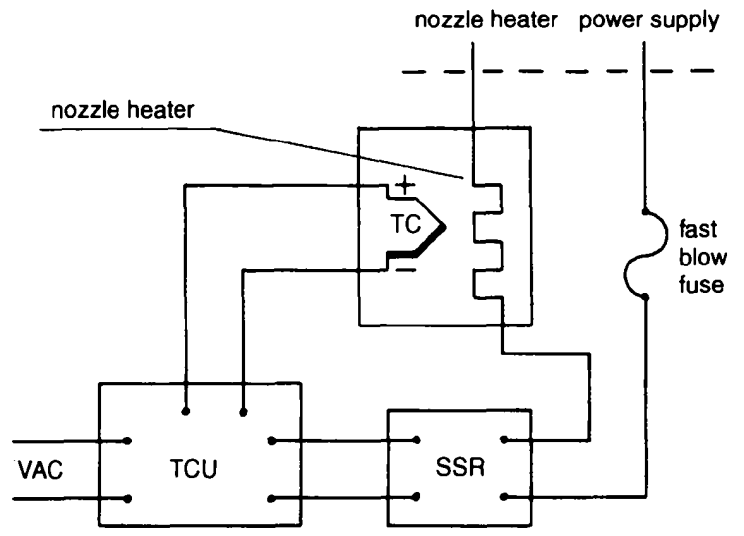

Fig. 4. Circuit diagram of the nozzle-heater temperature controller. TC $=$ thermocouple (copper-constantan; attached to the stainless-steel nozzle tip); TCU = temperature-control unit; $S S R=$ solid state relay. 
As the intensities of the standard reflections remain constant, the effect on the data collection is negligible. For measurements at temperatures below $153 \mathrm{~K}$ and for long-term data collections, the set-up of an enclosure is nevertheless strongly recommended.

In conclusion, the stationary nozzle attachment described offers an efficient supplement for a low-temperature device with standard coaxial nozzle assembly. The versatility of the diffractometer is considerably improved, permitting the choice of the system that is most adapted to the experimental needs. Various studies of compounds unstable at ambient conditions (species with a melting point down to $225 \mathrm{~K}$ were structurally investigated) have demonstrated the striking advantage of the stationary arrangement in combination with the oil-drop mounting technique. The low cost and little time required make the implementation particularly worthwhile.

The authors thank Dr Vincent Lynch (University of Texas at Austin) for helpful discussions. TK gratefully acknowledges a Feodor-Lynen scholarship by the Alexander von Humboldt
Foundation. The project was supported by the Deutsche Forschungsgemeinschaft, DFG (DS).

\section{References}

Abowitz, G. \& Ladell, J. (1968). J. Sci. Instrum. 2, 1, 113-117. Brock, C. P. \& Dunitz, J. D. (1990). Acta Cryst. B46, 795-806.

Cosier, J. \& Glazer, A. M. (1986). J. Appl. Cryst. 19, 105-107.

Hope, H. (1987). Experimental Organometallic Chemistry, ACS Symposium Series No. 357. Washington, DC: American Chemical Society.

Hope, H. (1988). Acta Cryst. B44, 22-26.

Koritsanszky, T, Buschmann, J., Luger, P., Knochel, A. \& Patz, M. (1994). J. Am. Chem. Soc. 116, 6748-6756.

Kottke, T. \& Stalke, D. (1993). J. Appl. Cryst. 26, 615-619.

Kottke, T. (1993). PhD thesis. University of Göttingen, Germany.

Luger, P. (1989). S. Afr. J. Chem. 42, 127-135.

Rudman, R. (1976). Low-Temperature X-ray Diffraction: Apparatus and Techniques. New York: Plenum.

Stalke, D. (1987). PhD thesis. University of Göttingen, Germany.

Stoe Diffraction Systems (1987). J. Appl. Cryst. 20, 449.

Strouse, C. D. (1976). Rev. Sci. Instrum. 47, 871-876.

Veith, M. \& Frank, W. (1988). Chem. Rev. 88, 81-92. 